\title{
Scope Dominance with Generalized Quantifiers
}

\author{
Gilad Ben-Avi and Yoad Winter
}

January 8, 2009

\begin{abstract}
When two quantifiers $Q_{1}$ and $Q_{2}$ satisfy the scheme $Q_{1} x Q_{2} y \phi \rightarrow Q_{2} y Q_{1} x \phi$, we say that $Q_{1}$ is scopally dominant over $Q_{2}$. This relation is central in analyzing and computing entailment relations between different readings of ambiguous sentences in natural language. This paper reviews the known results on scope dominance and mentions some open problems.
\end{abstract}

\section{Basic definitions}

An arbitrary generalized quantifier of signature $\left\langle n_{1}, \ldots, n_{k}\right\rangle$ over a non-empty domain $E$ is a relation $f \subseteq \wp\left(E^{n_{1}}\right) \times \ldots \times \wp\left(E^{n_{k}}\right)$, where $k \geq 1$, and $n_{i} \geq 1$ for all $i \leq k$ (e.g. Peters and Westerståhl, 2006, p.65). In short, we say that $f$ is a quantifier when it is of signature $\langle 1\rangle$, a determiner (relation) when it is of signature $\langle 1,1\rangle$, and a dyadic quantifier when it is of signature $\langle 2\rangle$. When $\mathcal{R}$ is a binary relation over some domain $\mathcal{E}$ (not necessarily $E$ ), we denote for every $\mathcal{X}, \mathcal{Y} \in \mathcal{E}:$

(1) $\quad$ a. $\mathcal{R}_{\mathcal{X}}=\{\mathcal{Y} \in \mathcal{E}: \mathcal{R}(\mathcal{X}, \mathcal{Y})\}$

b. $\mathcal{R}^{\mathcal{Y}}=\{\mathcal{X} \in \mathcal{E}: \mathcal{R}(\mathcal{X}, \mathcal{Y})\}$

In theories of natural language semantics, determiner relations are useful in describing the meaning of determiner expressions as in (2).

$$
\begin{array}{ll}
\text { every: } & \text { every }=\left\{\langle A, B\rangle \subseteq E^{2}: A \subseteq B\right\} \\
\text { some: } & \text { some }=\left\{\langle A, B\rangle \subseteq E^{2}: A \cap B \neq \emptyset\right\} \\
\text { more than half: } & \mathbf{m t h}=\left\{\langle A, B\rangle \subseteq E^{2}:|A \cap B|>|A \cap \bar{B}|\right\}
\end{array}
$$

It is well-known (Peters and Westerståhl, 2006, p.469) that meanings of natural language determiners - e.g. of the expression more than half - may be beyond what is expressible in first order logic.

We assume that nouns denote sets $A \subseteq E$. Noun phrase meanings are then described as in (3) using a quantifier $D_{A}$, where the noun denotation is the left 
argument of the determiner relation $D($ cf. (1a)).

(3)

$$
\begin{array}{ll}
\text { every student: } & \text { every }_{S}=\{B \subseteq E: S \subseteq B\} \\
\text { some teacher: } & \text { some }_{T}=\{B \subseteq E: T \cap B \neq \emptyset\} \\
\text { more than half of the students: } & \operatorname{mth}_{S}=\{B \subseteq E:|S \cap B|>|S \cap \bar{B}|\}
\end{array}
$$

Truth values of simple sentences with intransitive verbs are derived as in (4), using the membership statement that the set denotation of the verb is in the quantifier denotation of the subject, or, equivalently, that the pair of sets denoted by the noun and the verb are in the determiner relation.

(4)

$$
\begin{array}{ll}
\text { every student smiled: } & S M \in \text { every }_{S} \Leftrightarrow\langle S, S M\rangle \in \text { every } \Leftrightarrow \\
& S \subseteq S M \\
\text { some teacher cried: } & C \in \text { some }_{T} \Leftrightarrow\langle T, C\rangle \in \text { some } \Leftrightarrow \\
& T \cap C \neq \emptyset
\end{array}
$$

The iteration of two quantifiers $Q_{1}$ and $Q_{2}$ is the dyadic quantifier $Q_{1}-Q_{2}$ defined in (5). Iteration is used as in (6b) for describing meanings of simple sentences like $(6 \mathrm{a})$, with transitive verbs that denote binary relations.

(5) $Q_{1}-Q_{2} \stackrel{\text { def }}{=}\left\{R \subseteq E^{2}:\left\{x \in E: R_{x} \in Q_{2}\right\} \in Q_{1}\right\}$

(6) a. some teacher praised every student

b. $P \in$ some $_{T}-$ every $_{S} \Leftrightarrow$

$$
\left\{x \in E: P_{x} \in \text { every }_{S}\right\} \in \text { some }_{T} \Leftrightarrow
$$$$
T \cap\left\{x \in E: S \subseteq P_{x}\right\} \neq \emptyset
$$

The statement in (6b) is equivalent to the predicate calculus formula (7a). However, a well-known problem in linguistics (Ruys and Winter, 2008) is that transitive sentences like (6a) also have an "inverse scope" reading (7b).

(7) $\quad$ a. $\exists x[T(x) \wedge \forall y[S(y) \rightarrow P(x, y)]]$

b. $\forall y[S(y) \rightarrow \exists x[T(x) \wedge P(x, y)]]$

A way to use (generalized) quantifiers $Q_{1}$ and $Q_{2}$ for deriving the meaning of formula $(7 \mathrm{~b})$, is to define an operator ' $\sim$ ' of inverse iteration. The dyadic quantifier $Q_{1} \sim Q_{2}$ defined in (8) is used in (9b) for obtaining an alternative analysis of $(9 \mathrm{a})(=(6 \mathrm{a}))$.

(8) $Q_{1} \sim Q_{2} \stackrel{\text { def }}{=} \quad\left\{R^{-1}: R \in Q_{2}-Q_{1}\right\}$

$$
=\quad\left\{R \subseteq E^{2}:\left\{y \in E: R^{y} \in Q_{1}\right\} \in Q_{2}\right\}
$$

(9) a. some teacher praised every student

b. $P \in$ some $_{T} \sim$ every $_{S} \Leftrightarrow$

$\left\{y \in E: P^{y} \in\right.$ some $\left._{T}\right\} \in$ every $_{S} \Leftrightarrow$

$S \subseteq\left\{y \in E: T \cap P^{y} \neq \emptyset\right\}$ 
A trivial fact of first order logic is the entailment $(7 a) \Rightarrow(7 b)$. In natural language semantics, this is reflected in the logical relation between the two readings of sentences like (9a). To describe the general phenomenon, we define a notion of scope dominance (in short, "dominance") between quantifiers.

Definition 1 A quantifier $Q_{1}$ is scopally dominant over a quantifier $Q_{2}$, if $Q_{1}-Q_{2} \subseteq Q_{1} \sim Q_{2}$.

In cases of determiners $D, D^{\prime}$, where the quantifier $D_{A}$ is dominant over $D_{B}^{\prime}$ for any two sets $A$ and $B$, we say that $D$ is dominant over $D^{\prime}$. For example, the classical entailment $(7 \mathrm{a}) \Rightarrow(7 \mathrm{~b})$ amounts to the fact that for all $S, T \subseteq E$, the quantifier some so $_{S}$ is dominant over every $\mathbf{y}_{T}$. In short: some is dominant over every. Furthermore, as can be easily verified, some is also dominant over $\mathbf{m t h}$, where the latter quantifier is not first-order definable. The latter scope dominance is reflected in the relation between the two readings of sentence (10).

(10) A guard is standing in front of more than half of the churches.

The likely reading of sentence (10), in which more than half of the churches have a guard in front of them (potentially different guards), entails the less likely reading, in which more than half of the churches are associated with the same guard.

To describe such cases of scope dominance in natural languages, the general task is:

Characterize the quantifiers $Q_{1}, Q_{2}$ such that $Q_{1}$ is dominant over $Q_{2}$.

In this paper we give a review of previous results about this question, and point to some open problems.

\section{Results on scope dominance}

\subsection{Scope dominance and duality}

The complement $\bar{Q}$ of a quantifier $Q$ is the set $\wp(E) \backslash Q$, whereas $Q$ 's postcomplement is the set $Q-\stackrel{\text { def }}{=}\{A \subseteq E: E \backslash A \in Q\}$. The dual $Q^{d}$ of a quantifier $Q$ is the complement of $Q$ 's postcomplement:

$$
Q^{d} \stackrel{\text { def }}{=} \overline{(Q-)}=(\bar{Q})-=\{A \subseteq E: E \backslash A \notin Q\} .
$$

Obviously, these three relations between quantifiers are symmetric, and $(\bar{Q})^{d}=$ $Q-$. This is naturally described using figure 1, which generalizes the Aristotelian square of opposition.

Whenever two determiner relations $D$ and $D^{\prime}$ form complement (postcomplement, dual) quantifiers $D_{A}$ and $D_{A}^{\prime}$ on every set $A$, we say that the determiners are each other's complements (postcomplements, duals) and write that $D^{\prime}=\bar{D}$ ( $D^{\prime}=D-, D^{\prime}=D^{d}$, respectively). The classical square of opposition is between the four determiner expressions every, some, no and not every. More 


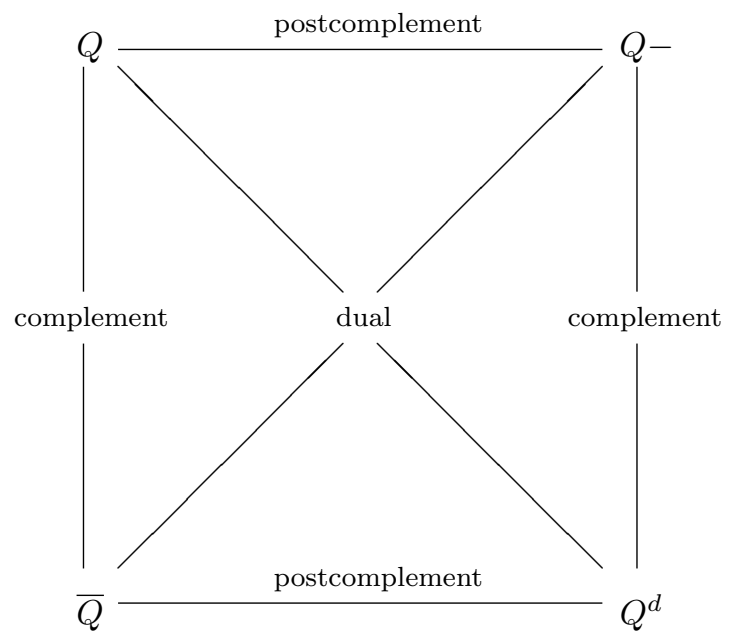

Figure 1: The Square of Opposition

generally, the opposition holds between the following determiners, which form the classical square in the special case $n=0$.

(11)

$$
\begin{aligned}
& D=\text { all_but_at_most_n } \quad=\left\{\langle A, B\rangle \subseteq E^{2}:|A \backslash B| \leq n\right\} \\
& D^{d}=\text { more_than } \_n=\left\{\langle A, B\rangle \subseteq E^{2}:|A \cap B|>n\right\} \\
& D-=\text { at_most } \_n=\left\{\langle A, B\rangle \subseteq E^{2}:|A \cap B| \leq n\right\} \\
& \bar{D}=\operatorname{not}(\text { all_but_at_most_n })=\left\{\langle A, B\rangle \subseteq E^{2}:|A \backslash B|>n\right\}
\end{aligned}
$$

The relation between quantifier duality and scope dominance is described in the following fact (Westerståhl, 1986, p.278).

Fact 1 For all quantifiers $Q_{1}$ and $Q_{2}: Q_{1}$ is scopally dominant over $Q_{2}$ iff $Q_{2}^{d}$ is dominant over $Q_{1}^{d}$.

For instance, just like some (=more_than_0) is dominant over more_than_3, so is all_but_at_most_3 dominant over every (=all_but_at_most_0).

\subsection{Special cases of scope dominance}

Two different special cases of scope dominance were studied by Zimmermann (1993) and Westerståhl (1996). Zimmermann characterizes the class of scopeless quantifiers: those quantifiers $Q$ that satisfy for all $Q_{1} \subseteq \wp(E): Q-Q_{1}=Q \sim Q_{1}$. He shows that the scopeless quantifiers over $E$ are precisely the principal ultrafilters over $E$ : the quantifiers $\{B \subseteq E: a \in B\}$ for some arbitrary $a \in E .^{1}$

\footnotetext{
${ }^{1}$ Zimmermann characterizes scopelessness in a more general case, where $Q$ and $Q_{1}$ are not necessarily defined over the same domain. The property we mention here is a direct result of his characterization.
} 
Westerståhl (1996) characterizes the class of self-commuting quantifiers: those quantifiers $Q$ that satisfy $Q-Q=Q \sim Q$. He shows that $Q$ is self-commuting iff $Q$ is either a union or an intersection of principal ultrafilters, or a finite symmetric difference of principal ultrafilters, or a complement of such a symmetric difference.

Clearly, the notion of scope dominance is more general than scopelessness or self-commutativity: a quantifier $Q$ is scopeless iff $Q$ and $Q^{d}$ are both dominant over any quantifier, while $Q$ is self-commuting iff it is dominant over itself. However, it should be noted that the results on scope dominance that we survey below do not fully subsume these results by Zimmermann and Westerståhl, which hold irrespectively of quantifier monotonicity and the cardinality of the domain.

\subsection{Characterizing scope dominance}

A quantifier $Q$ over $E$ is called upward (downward) monotone if $A \subseteq B \subseteq E$ implies that $B \in Q$ if (only if) $A \in Q$. A determiner $D$ over $E$ is upward (downward) monotone if $D_{A}$ is upward (downward) monotone for all $A \subseteq E$.

Westerståhl (1986) characterizes the pairs $D_{1}$ and $D_{2}$ of upward monotone determiners for which $D_{1}$ is dominant over $D_{2}$. He shows that over finite domains these are precisely the cases where $D_{1}$ is some or $D_{2}$ is every. Westerståhl's characterization is stated in a more general form in the following theorem. $^{2}$

Theorem 2 Let $Q_{1}$ and $Q_{2}$ be two nontrivial upward monotone quantifiers over a finite domain $E$. Then $Q_{1}$ is scopally dominant over $Q_{2}$ iff $Q_{1}=\mathbf{s o m e}_{A}$ or $Q_{2}=$ every $_{A}$ for some $A \subseteq E$.

In general, however, this characterization is too narrow for infinite domains. For instance, the quantifier $Q_{1}=\{X \subseteq \mathbb{N}:\{1,2\} \subseteq X\}$ is dominant over the quantifier $Q_{2}=\{X \subseteq \mathbb{N}:|Y \backslash X| \in \mathbb{N}\}$. This is reflected in the logical relation between the two readings of sentence (12). But there is no $A \subseteq \mathbb{N}$ such that $Q_{1}=$ some $_{A}$ or $Q_{2}=\operatorname{every}_{A}$.

(12) Both item 1 and item 2 cover all but finitely many cases.

Altman et al. (2005) extend Westerståhl's result for upward monotone quantifiers over countable domains. ${ }^{3}$ For the formulation of the characterization, they define a property of quantifiers called the Descending Chain Condition (DCC). A quantifier $Q$ is said to satisfy (DCC) if for every descending sequence $A_{1} \supseteq A_{2} \supseteq \cdots A_{n} \supseteq \cdots$ in $Q$, the intersection $\bigcap_{i} A_{i}$ is in $Q$ as well. For instance, any quantifier of the form every ${ }_{A}$ satisfies (DCC), while a quantifier some $_{A}$ satisfies (DCC) if and only if $A$ is finite. The following theorem by Altman et al. is a generalization of Theorem 2 to countable domains.

\footnotetext{
${ }^{2}$ In fact, Westerståhl's result is restricted to "global" determiner functors (abstracting over the domain $E$ ), which are furthermore logical, i.e. satisfy the familiar restrictions of conservativity, permutation invariance and extension.

${ }^{3}$ See Altman et al. (2001) for an earlier, and more restricted, characterization of scope dominance for the same class of quantifiers.
} 
Theorem 3 Let $Q_{1}$ and $Q_{2}$ be upward monotone quantifiers over a countable domain $E$. Then $Q_{1}$ is scopally dominant over $Q_{2}$ iff all of the following requirements hold:

(i) $Q_{1}^{d}$ or $Q_{2}$ are closed under finite intersections;

(ii) $Q_{1}^{d}$ or $Q_{2}$ satisfy (DCC);

(iii) $Q_{1}^{d}$ or $Q_{2}$ are not empty.

Note that, on finite domains, an upward monotone quantifier $Q$ is closed under intersections if and only if it is of the form every ${ }_{A}$. Furthermore, over finite domains, (ii) is a trivial consequence of (i). So this is indeed a generalization of Theorem 2.

If we consider not only upward but also downward monotone quantifiers, then also on finite domains there are instances of scope dominance that do not involve quantifiers of the form every ${ }_{A}$ or some $_{A}$. For instance, the logical relation between the two readings of sentence (13) is a manifestation of the scope dominance relation between $\mathbf{m t h}(\mathrm{cf} .(2))$ and no = at_most_0 (cf. (11)).

(13) More than half of the teachers praised no student.

Theorem 4 below from Ben-Avi and Winter (2004), together with its dual, provide a characterization over finite domains of scope dominance between quantifiers of "opposite" (upward/downward) monotonicities. In this theorem we use the notion of a minimal set in a quantifier. Standardly, we say that a set $X \in Q$ is minimal in $Q$ if $Y \subsetneq X$ implies $Y \notin Q$.

Theorem 4 Let $Q_{1}$ and $Q_{2}$ be two (non-trivial) quantifiers over a finite domain $E$, s.t. $Q_{1}$ is upward monotone and $Q_{2}$ is downward monotone. Let

$$
n \stackrel{\text { def }}{=} \max \left\{|X|: X \text { is minimal in } \overline{Q_{2}}\right\}
$$

Then $Q_{1}$ is scopally dominant over $Q_{2}$ iff every $Q \subseteq Q_{1}$ with $|Q| \leq n+1$ has a nonempty intersection (i.e., $\cap Q \neq \emptyset$ ).

Theorem 4 captures the dominance in (13). For more examples of this sort see Ben-Avi and Winter (2004).

\subsection{Inverse linking constructions}

One of the puzzling structures for theories of quantifier scope in natural language involves sentences like the following, which are sometimes referred to as inverse linking constructions.

(14) Some student from every city participated.

In Predicate Calculus notation, the prominent reading of (14) is (15b), whereas a possible but less plausible reading is (15a). 
a. $\exists x[S(x) \wedge \forall y[C(y) \rightarrow F(x, y)] \wedge P(x)]$

("there exists a student who is from every city, and that student participated")

b. $\forall y[C(y) \rightarrow \exists x[S(x) \wedge F(x, y) \wedge P(x)]]$

("for every city $y$, there exists a student who is from $y$ and who participated")

As a matter of syntactic structure, inverse linking sentences involve a noun phrase that appears within the left argument of a determiner expression, where this argument is further restricted by a noun. In the case of sentence (14), the noun phrase every city is in the left argument of the determiner expression some, restricted by the noun student. To capture the effect we call "restriction", we adopt the following notation for any determiner over $E$ and a set $X \subseteq E$.

$$
\begin{aligned}
& \text { a. } D: X=\{\langle A, B\rangle:\langle A \cap X, B\rangle \in D\} \\
& \text { b. } D^{: X}=\{\langle A, B\rangle:\langle A, B \cap X\rangle \in D\}
\end{aligned}
$$

The denotations in inverse linking sentences like (14) involve two determiners $D$ and $D^{\prime}$, three sets $A, B$ and $P$, and a binary relation $R$. In the example, the determiners correspond to some and every, the sets correspond to student, city and participated, and the binary relation corresponds to from. Using the "restriction" notation in (16), the two readings of inverse linking constructions are expressed as follows.
a. $R \in D_{: A}^{P}-D^{\prime}{ }_{B}$
b. $R \in D_{: A}^{P} \sim D^{\prime}{ }_{B}$

It is easy to verify that the two predicate logic formulae in (15) are equivalent to the following claims.

$$
\begin{aligned}
& \text { a. } F \in \text { some }_{: S}^{P}-\text { every }_{C} \\
& \text { b. } F \in \text { some }_{: S}^{P} \sim \text { every }_{C}
\end{aligned}
$$

The intersectivity (cf. Peters and Westerståhl, 2006, p.210) of the determiner some implies that some $e_{: S}^{P}=$ some $^{S \cap P}$. As a result, the familiar dominance of some over every accounts for the dominance of some $e_{S}^{P}$ over every ${ }_{C}$, or the entailment from (18a) $(=(15 a))$ to $(18 b)(=(15 b))$.

Matters get more involved when the first determiner in the inverse linking construction is not intersective. Consider sentence (19) and its two analyses in (19a-b).

(19) Every student from some city participated.
a. $F \in$ every $_{: S}^{P}-$ some $_{C}$
b. $F \in$ every $_{: S}^{P} \sim$ some $_{C}$ 
Two facts should be noted about the quantifier $Q=$ every: $_{: S}^{P}$ in these analyses. First, the effect of supplying the right argument $P$ of the determiner every is that in general $Q$ is not an upward monotone quantifier. Second, for any sets $S$ and $P$, the quantifier $Q$ is furthermore downward monotone, as a result of the simple fact $Q=$ every: $:_{A}^{P}=$ every $^{P \cup A}$, and the downward monotonicity of every in its left argument. ${ }^{4}$ Consequently, we can use Theorem 4 to show that every: $_{: S}^{P}$ is dominant over some s , unless $C=\emptyset$. In fact, the dual of some , $_{C}$ every $_{C}$, is upward monotone, and $\bigcap$ every $_{C} \neq \emptyset$, unless $C=\emptyset$. By Theorem 4 , every $_{C}$ is dominant over any downward monotone quantifier; ${ }^{5}$ specifically, it is dominant over the dual of every ${ }_{: S}^{P}$. By Fact 1 , every $P$ : ${ }_{S}$ is dominant over some $_{C}$.

We conclude that the scheme in (17) allows us to use the theorems above, which are stated in terms of quantifiers, for characterizing at least some of the entailments between readings of inverse linking constructions, which are initially stated in terms of determiners. Further study of the way to characterize such entailments must wait for further research.

\subsection{Open questions}

In addition to the characterization of entailments between readings, results about scope dominance itself can be extended and generalized in some directions. This includes a full characterization of scope dominance between arbitrary quantifiers, and over domains with arbitrary cardinality. At present there are no such general results known to us.

\section{Discussion}

Nissim Francez (p.c.) has expressed some doubts about the importance of scope dominance for theoretical and computational semantics of natural language. As always, we are happy to differ. Although it is a "purely" combinatorial problem, it seems clear that a full characterization of scope dominance would deepen our understanding of ambiguity in natural language. It is a fact that some sentences show intricate logical relations between readings that grammarians find necessary to assume. This fact has led to considerable confusion in the theoretical linguistic literature (Ruys, 2002). Having sound rules of thumb for the situations in which such relations may appear could undoubtedly help to prevent further descriptive inadequacies. Furthermore, some works (Chaves, 2003; Altman and Winter, 2005) have started to explore the possibilities of computing scope dominance with natural language sentences. As Francez has

\footnotetext{
${ }^{4}$ More generally, the presentation $D_{: A}^{P}=D^{P \cup \bar{A}}$ follows for any co-intersective determiner $D$. Co-intersectivity requires that if $A \backslash B=A^{\prime} \backslash B^{\prime}$, then $B \in D_{A} \Leftrightarrow B^{\prime} \in D_{A^{\prime}}$. (Keenan, 2006)

${ }^{5}$ It should be noted that Theorem 4 as stated here does not cover trivial quantifiers. However, $C \neq \emptyset$ implies that every $_{C}$ is not trivial, in which case it is easy to verify that it is dominant over any trivial quantifier.
} 
argued, at this point it is too early to know if such algorithms can be useful for reasoning under ambiguity (e.g. Reyle, 1995; Van Deemter, 1996; Van Eijck and Jaspars, 1996). However, this is one domain where non-trivial problems about inference in natural language seem tractable. We therefore believe that scope dominance introduces an interesting challenge for a realm in which Francez (Fyodorov et al., 2003; Zamansky et al., 2006; Francez and Dyckhoff, 2007) has continuously contributed.

\section{References}

Altman, A. and Winter, Y. (2005). Computing dominant readings with upward monotone quantifiers. Research on Language and Computation, 3, 1-43.

Altman, A., Keenan, E., and Winter, Y. (2001). Monotonicity and relative scope relations. In R. van Rooy and M. Stokhof, editors, Proceedings of the 13th Amsterdam Colloquium, pages 25-30.

Altman, A., Peterzil, Y., and Winter, Y. (2005). Scope dominance with upward monotone quantifiers. Journal of Logic, Language and Information, 14, 445455.

Ben-Avi, G. and Winter, Y. (2004). Scope dominance with monotone quantifiers over finite domains. Journal of Logic, Language and Information, 13, 385402.

Chaves, R. P. (2003). Non-redundant scope disambiguation in underspecified semantics. In B. ten Cate, editor, Proceedings of the 8th ESSLLI student session, pages 47-58.

Francez, N. and Dyckhoff, R. (2007). Proof-theoretic semantics for natural language. In Mathematics of Language (MOL) 10.

Fyodorov, Y., Winter, Y., and Francez, N. (2003). Order-based inference in natural logic. Logic Journal of the IGPL, 11, 385-417.

Keenan, E. L. (2006). Quantifiers: Semantics. In K. Brown, editor, Encyclopedia of Language and Linguistics, Vol. 10. Elsevier, Oxford.

Peters, S. and Westerståhl, D. (2006). Quantifiers in Language and Logic. Clarendon Press, Oxford.

Reyle, U. (1995). On reasoning with ambiguities. In Proceedings of the EACL'95.

Ruys, E. (2002). Wide scope indefinites: genealogy of a mutant meme. Unpublished ms., Utrecht University. Downloadable from http://www.let.uu.nl/ $\sim$ Eddy.Ruys/personal/download/indefinites.pdf. 
Ruys, E. and Winter, Y. (2008). Quantifier scope in formal linguistics. To appear in D. Gabbay (ed.), Handbook of Philosophical Logic - Second Edition.

Van Deemter, K. (1996). Towards a logic of ambiguous expressions. In K. van Deemter and S. Peters, editors, Semantic Ambiguity and Underspecification, pages 203-237. Cambridge University Press, Cambridge, England.

Van Eijck, D. J. N. and Jaspars, J. O. M. (1996). Ambiguity and Reasoning. Technical report, Computer Science / Department of Software Technology, Centrum voor Wiskunde en Informatica (CWI), CS-R9616.

Westerståhl, D. (1986). On the order between quantifiers. In M. Furberg et al., editors, Acta Universitatis Gothoburgensis, pages 273-285. Göteborg University.

Westerståhl, D. (1996). Self-commuting quantifiers. The Journal of Symbolic Logic, 61, 212-224.

Zamansky, A., Winter, Y., and Francez, N. (2006). A 'natural logic' inference system using the lambek calculus. Journal of Logic, Language and Information, 15, 273-295.

Zimmermann, T. E. (1993). Scopeless quantifiers and operators. Journal of Philosophical Logic, 22, 545-561. 